\title{
Erratum to: Vascular access registry of Serbia: a 4-year experience
}

\author{
Tamara Jemcov ${ }^{1,2}$ - Nada Dimkovic ${ }^{1,3}$ • Dragan Jovanovic ${ }^{4}$. Tanja Lazarevic ${ }^{5}$. \\ Igor Mitic $^{6} \cdot$ Radomir Naumovic $^{1,7} \cdot$ Sanja Simic-Ogrizovic $^{1,7} \cdot$ Radmila Velickovic $^{8}$. \\ Vascular Access Study Group
}

Published online: 25 January 2017

(c) Springer Science+Business Media Dordrecht 2017

\section{Erratum to: Int Urol Nephrol DOI 10.1007/s11255-016-1378-9}

Authors want to correct the list of authors by expanding the number of coauthors and by including all contributors in the Vascular Access Study Group.

Vascular Access Study Group (in alphabetic order):

Andric Branislav, Antic Miodrag, Aracki Snezana, Arsenovic Aleksandra, Berto Sabo Anika, Bogdanovic Jasmina, Cekovic Biljana, Cuckovic Cedomir, Cukic Zoran, Cveticanin Anica, Djordjevic Verica, Dudic Svetlana, Gajic Snezana, Gojakovic Biljana, Golubovic Predrag, Gucic Ljubinka, Hadzibulic Edvin, Hadzifejzovic Mersada, Hamzagic Nedim, Haviza-Lilic Branimir, Ilic Mira, Ilic
Nasta, Jelacic Rosa, Kostic Mirjana, Kovacevic Miodrag, Lazarevic Tatjana, Markovic Rodoljub, Micunovic Vesna, Milenkovic Olgica, Milenkovic Radomir, Milenkovic Srboljub, Milicevic Biserka, Milicevic Olivera, Nikolic Zora, Obrenovic Slavica, Orescanin Mira, Pavlovic Stevan, Pesic Snezana, Petkovic Dobrila, Pilipovic Dragana, Prokopovic Miomir, Radovanovic Zoran, Rakic Nenad, Rangelov Vanja, Sefer Kornelija, Sibalic Simin Marija, Stefanovic Nikola, Stojanovic Dragoslav, Stojanovic Stanojevic Marina, Tirmenstajn Jankovic Biserka, Vasic Jovanovic Vesna, Vasilic Kokotovic Olivera, Vojinovic Goran, Vuckovic Dragana, Vukelic Vesna, Vukic Jasmina, Zagorac Nikola, Zec Nenad.

The online version of the original article can be found under doi:10.1007/s11255-016-1378-9.

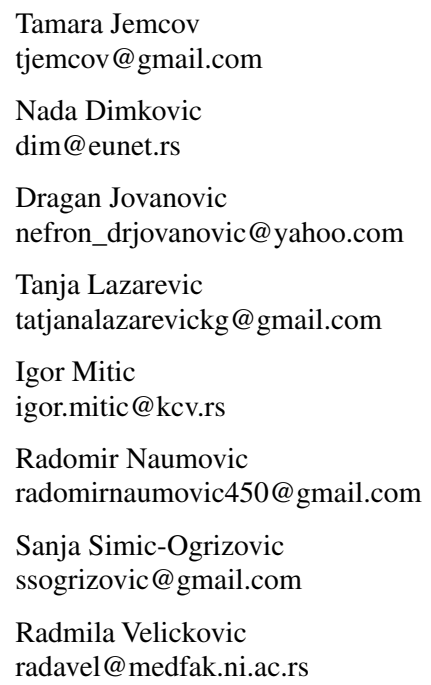

School of Medicine, University of Belgrade, Belgrade, Serbia

2 Department of Nephrology, Clinical Hospital Center Zemun, Belgrade, Serbia

3 Clinical Department for Nephrology and Dialysis, Zvezdara University Medical Center, Belgrade, Serbia

4 Military Academy, Belgrade, Serbia

5 Department of Nephrology, Clinical Center Kragujevac, Kragujevac, Serbia

6 Clinic for Nephrology and Immunology, Clinical Center Novi Sad, Novi Sad, Serbia

7 Clinic for Nephrology, Clinical Center Serbia, Belgrade, Serbia

8 Clinic for Nephrology, Clinical Center Nis, Nis, Serbia 